\title{
Fatores associados a recusa do exame citopatológico por mulheres atendidas em Unidades Básicas de Saúde no Brasil
}

\author{
Factors associated with the refusal of Pap smears by women attending Basic Health Units in Brazil \\ Factores asociados al rechazo de Papanicolaou por mujeres que asisten a Unidades Básicas de
} Salud en Brasil

Recebido: 09/11/2021 | Revisado: 19/11/2021 | Aceito: 23/11/2021 | Publicado: 04/12/2021

Milene Manoelly Dutra Silvestre Marques
ORCID: https://orcid.org/0000-0001-9403-5850
Centro Universitário Maurício de Nassau, Brasil
E-mail: milenemds15@gmail.com
Rayane Elias da Silva Barcelos Pedrozo
ORCID: https://orcid.org/0000-0001-8313-6517
Centro Universitário Maurício de Nassau, Brasil
E-mail: rayanebarcelos16@ hotmail.com

\begin{abstract}
Resumo
Objetivo: Identificar, através da literatura pertinente, os principais motivos pelo qual o exame citológico ainda possui baixa procura na Atenção Básica. Método: A pesquisa consiste numa Revisão Integrativa da Literatura, com abordagem qualitativa e do tipo exploratória. Resultados: O câncer de colo de útero é diagnosticado através do exame citopatológico. Existem vários fatores que dificultam a realização do exame citológico para mulheres a procurar o exame regularmente é necessário aumentar a oferta de vagas do mesmo para facilitar a marcação, assim como realizar atividades educativas é de extrema importância. Conclusão: compreende-se que existem diversos motivos pelos quais dificultam a adesão das mulheres ao exame citopatológico, porém cabe a equipe de saúde sensibilizar a mulher quanto a sua importância, enfatizando os benefícios de detectar precocemente um câncer de colo de útero para um prognóstico favorável e redução na morbimortalidade.
\end{abstract}

Palavras-chave: Citologia; Cuidados de enfermagem; Esfregaço vaginal; Políticas públicas de saúde; Educação em saúde.

\begin{abstract}
Objective: To identify, through the pertinent literature, the main reasons why the cytological examination still has low demand in Primary Care. Method: The research consists of an Integrative Literature Review, with a qualitative and exploratory approach. Results: Cervical cancer is diagnosed through cytopathological examination. There are several factors that make it difficult to carry out the cytological exam for women to seek the exam regularly, it is necessary to increase the number of places available to facilitate the appointment, as well as carrying out educational activities is extremely important. Conclusion: it is understood that there are several reasons why it is difficult for women to adhere to the Pap smear test, but it is up to the health team to make women aware of its importance, emphasizing the benefits of early detection of cervical cancer for a favorable prognosis and reduction in morbidity and mortality. Keywords: Cytology; Nursing care; Vaginal smear; Health policy; Health education.
\end{abstract}

\section{Resumen}

Objetivo: Identificar, a través de la literatura pertinente, las principales razones por las que el examen citológico aún tiene baja demanda en Atención Primaria. Método: La investigación consiste en una Revisión Integrativa de la Literatura, con un enfoque cualitativo y exploratorio. Resultados: El cáncer de cuello uterino se diagnostica mediante examen citopatológico. Son varios los factores que dificultan la realización del examen citológico para que la mujer solicite el examen de forma regular, es necesario aumentar la cantidad de plazas disponibles para facilitar la cita, además de realizar actividades educativas es sumamente importante. Conclusión: se entiende que son varios los motivos por los que es difícil que las mujeres se adhieran a la prueba de Papanicolaou, pero le corresponde al equipo de salud concienciar a las mujeres de su importancia, enfatizando los beneficios de la detección precoz del cáncer cervicouterino para un pronóstico favorable y una reducción de la morbilidad y la mortalidad. Palabras clave: Citología; Cuidado de enfermera; Hisopo vaginal; Políticas de salud pública; Educación para la salud. 


\section{Introdução}

As Unidades Básicas de Saúde (UBS) são conhecidas como a porta de entrada preferencial para o sistema de saúde, onde deve ser ofertado diversos serviços para uma assistência à saúde universal, integral e equitativa, como recomenda os princípios e diretrizes do Sistema Único de Saúde (SUS). As políticas públicas são cada vez mais estudadas com o objetivo de atender a necessidade da população e suas particularidades, através de ações educativas e busca ativa para fazer com que a comunidade procure os serviços da Atenção Básica (AB) (Aguilar; Soares, 2015; Brasil, 2017).

$\mathrm{O}$ câncer do colo do útero (CCU) é uma doença que acomete mulheres residentes em países desenvolvidos e em desenvolvimento, mas sua incidência é maior em países em desenvolvimento. No Brasil se apresenta como a segunda neoplasia diagnóstica em mulheres, por isso o CCU é visto como um importante problema de saúde pública no país. Este tipo de câncer pode ser detectado e tratado precocemente através da realização do exame citopatológico, também conhecido como preventivo ou Papanicolau. É prático, de baixo custo e muito eficaz, realizado a partir do esfregaço de células da ectocérvice e da endocérvice que serão coletados do colo do útero e enviados ao laboratório para análise das células (Aguilar; Soares, 2015; Discacciati et al., 2014; Oliveira et al., 2014).

Nos países em desenvolvimento precisa-se de incitação para cobertura do Papanicolau. Segundo as diretrizes, no Brasil o rastreamento do CCU é necessário ser executado anualmente em mulheres com faixa etária de 25 a 64 anos ou que tenha iniciado a vida sexual, após dois exames seguidos com resultado normal, em um intervalo de um ano, o exame pode ser realizado a cada três anos. Esse rastreamento é essencial para identificar as mulheres que não realizaram o citopatológico por falta de conhecimento ou por não querer fazer o exame, podendo iniciar ações de educação em saúde para conscientizá-las da importância (Brasil, 2013).

Os fatores de risco para desenvolvimento do CCU são: início precoce de atividade sexual, sexo desprotegido, vários parceiros sexuais, tabagismo, uso prolongado de anticoncepcionais, higiene, exposição a Doenças Sexualmente Transmissível (DST) e contato com o Papiloma Vírus Humano (HPV). O CCU tem um crescimento lento e silencioso, sendo assim, quanto mais precoce o diagnóstico mais eficaz o tratamento e redução na taxa de incidência (Discacciati et al., 2014).

Existem diversos tabus para realização desse exame, como: medo, insegurança, pouco conhecimento sobre a patologia, difícil acesso, condições culturais e socioeconômicas. Desta forma ações de promoção e prevenção podem ser implantadas pela equipe multidisciplinar da UBS, de forma que a informação seja transmitida de maneira didática, garantindo que todas as mulheres compreendam e tomem consciência da imprescindibilidade da realização anual ou conforme indicação profissional do exame citopatológico (Rodrigues et al., 2020).

A questão norteadora da pesquisa é definida pela indagação: Quais barreiras encontradas para a não realização da citologia na UBS?

Diante do exposto, este artigo tem como objetivo identificar, através da literatura pertinente, os principais motivos pelo qual o exame citológico ainda possui baixa procura na Atenção Básica.

\section{Metodologia}

A presente pesquisa trata-se de uma Revisão Integrativa da Literatura, com abordagem qualitativa, do tipo exploratória. Este tipo de pesquisa permite resumir os conhecimentos encontrados, assim como permite a análise crítica dos resultados relevantes de cada estudo, sendo importante dar prioridade a literatura mais recente para um melhor embasamento teórico científico sobre o tema proposto (Soares et al., 2014).

O processo de elaboração desta pesquisa seguiu as seguintes fases de elaboração: Definição da pergunta condutora, seleção da literatura que compuseram a amostra do estudo; análise dos textos incluídos na revisão; interpretação dos resultados e discussão dos achados. Para a pesquisa desses estudos na rede eletrônica, utilizou-se as bases de dados: Scientific Electronic 
Library Online (SCIELO), Biblioteca Virtual em Saúde (BVS) e Bireme. Os descritores foram retirados dos Descritores em Ciências da Saúde (DeCs), sendo: Citologia; Cuidados de Enfermagem; Esfregaço Vaginal; Políticas Públicas de Saúde; Educação em Saúde, utilizando os operadores boleanos "and" e "or".

Para a construção deste artigo, foi formulada a pergunta condutora: Quais barreiras encontradas para a não realização da citologia na UBS? E a partir disso, buscaram-se diversos artigos que englobasse o tema proposto para fazer a inclusão nos resultados e discussão e assim obter uma revisão de literatura baseada em estudos publicados na rede eletrônica.

Segue abaixo no Quadro 1 para melhor elucidação e entrosamento, os critérios de inclusão e exclusão que foram definidos e escolhidos de acordo com os objetivos do presente artigo, para que desta forma os mesmos sejam elucidados e alcançados.

Quadro 1. Critérios de inclusão e exclusão.

\begin{tabular}{|l|l|}
\hline Critérios de Inclusão & Critérios de Exclusão \\
\hline Artigos disponíveis eletronicamente. & Editoriais. \\
Artigos completos. & Resumos. \\
Publicados em português, inglês ou espanhol. & Anais. \\
Estudos com abordagem qualitativa e quantitativa. & Comentários. \\
Estudos que abordaram ações de educação em saúde. & Opiniões. \\
Estudos que se apresentaram dentro da população & Pesquisas publicadas sem o parecer de um Comitê de \\
avaliada. & Ética em Pesquisa envolvendo seres humanos. \\
Manuais do Ministério da Saúde & Artigos repetidos em uma ou mais bases de dados \\
& citados. \\
\hline
\end{tabular}

Fonte: Autores.

Após o cruzamento dos descritores, os artigos encontrados foram submetidos à análise dos critérios de exclusão. Além disso, foi executada uma leitura criteriosa dos resumos a fim de observar os que atendem aos objetivos da pesquisa. Os artigos pré-selecionados foram lidos na íntegra, eliminando aqueles que não atendem aos critérios de inclusão. As evidências científicas das publicações foram categorizadas por tipo de estudo, sujeitos participantes, resultados significativos encontrados e conclusões dos autores.

\section{Resultados e Discussão}

Após a pesquisa nas bases de dados foram achados 134 artigos, destes utilizamos 17, seguindo os princípios estabelecidos nos critérios de inclusão e exclusão. Os artigos incluídos, nesta revisão, estão abaixo descritos conforme: o nome do periódico, título do artigo e autor (es) no Quadro 2, cenário da pesquisa, metodologia aplicada e ano da publicação no Quadro 3, objetivos e resultados no Quadro 4. 
Quadro 2. Resumo dos estudos pesquisados envolvendo periódico, título e autor.

\begin{tabular}{|c|c|c|}
\hline Períodico & Título & Autor \\
\hline Cad. Saúde Coletiva & $\begin{array}{l}\text { Fatores associados à realização de } \\
\text { citologia para prevenção de câncer de } \\
\text { colo uterino em uma comunidade de } \\
\text { baixa renda }\end{array}$ & $\begin{array}{l}\text { Falcão, G. B., Ibiapina, F. L. P., } \\
\text { Feitosa, H. N., Feitosa, T. S., } \\
\text { Lacerda, P. D., Braga, J. U., \& } \\
\text { Carvalho FHC. }\end{array}$ \\
\hline Revista enfermagem EERJ & $\begin{array}{l}\text { Conhecimento e prática das mulheres } \\
\text { atendidas na unidade de saúde da } \\
\text { família sobre o papanicolaou }\end{array}$ & $\begin{array}{l}\text { Andrade, M. S., Paixão, G. P. S., \& } \\
\text { Santiago, T. R. }\end{array}$ \\
\hline Revista Cuidarte & $\begin{array}{l}\text { Educação popular como instrumento } \\
\text { participativo para a prevenção do } \\
\text { câncer ginecológico: Percepção das } \\
\text { mulheres. }\end{array}$ & $\begin{array}{l}\text { Souza, K. R., Paixão, G. P. N., } \\
\text { Almeida, E. S., Sousa, A. R., Lirio, } \\
\text { J. G. S., \& Campos, L. M. }\end{array}$ \\
\hline $\begin{array}{l}\text { Revista de Enfermagem do } \\
\text { centro oeste mineiro }\end{array}$ & $\begin{array}{l}\text { Atividades educativas no controle } \\
\text { câncer de colo do útero: Relato de } \\
\text { experiência }\end{array}$ & $\begin{array}{l}\text { Valente, C. A., Andrade, V., } \\
\text { Soares, M. B. O., \& Silva, S. R. }\end{array}$ \\
\hline $\begin{array}{l}\text { Revista científica de } \\
\text { enfermagem. }\end{array}$ & $\begin{array}{l}\text { A importância da prevenção do câncer } \\
\text { do colo uterino: em pauta o exame de } \\
\text { Papanicolau. }\end{array}$ & $\begin{array}{l}\text { Santos, M. A., Audickas, R. C., } \\
\text { Coutinho, S. C., Silva, J., \& Souza, } \\
\text { L. N. }\end{array}$ \\
\hline Ministério da Saúde. & $\begin{array}{l}\text { Controle dos cânceres do colo do útero } \\
\text { e da mama. }\end{array}$ & Ministério da Saúde. \\
\hline Ciência \& Saúde Coletiva & $\begin{array}{l}\text { Fatores associados a não realização de } \\
\text { Papanicolau } \\
\text { em mulheres quilombolas. }\end{array}$ & $\begin{array}{l}\text { Oliveira, M. V., Guimarães, M. D. } \\
\text { C., \& França, E. B. }\end{array}$ \\
\hline Rev Saúde Pública & $\begin{array}{l}\text { Integralidade no cuidado ao câncer do } \\
\text { colo do útero: avaliação do acesso }\end{array}$ & $\begin{array}{l}\text { Silva, K. B., Bezerra, A. F. B., } \\
\text { Chaves, L. D. P., \& Tanaka, O. Y. }\end{array}$ \\
\hline $\begin{array}{l}\text { Anuário Pesquisa e Extensão } \\
\text { Unoesc Xanxerê. }\end{array}$ & Exame Citopatológico. & $\begin{array}{l}\text { Salvi, E. S. F., Pompermaier, C., } \\
\text { Floriani, F., Barrionuevo, V., \& } \\
\text { Block, J. C. }\end{array}$ \\
\hline Revista Intersaúde & $\begin{array}{l}\text { A importância do exame citopatológico } \\
\text { nas Unidades Básicas de Saúde: A } \\
\text { atuação do Enfermeiro. }\end{array}$ & Lopes, P., \& Lopes, A. \\
\hline $\begin{array}{l}\text { Brazilian Journal of } \\
\text { Development. }\end{array}$ & $\begin{array}{l}\text { Training of primary care nurses } \\
\text { regarding cervical cytopathological } \\
\text { examination. }\end{array}$ & $\begin{array}{l}\text { Silva, N. F. C. P., Silva, C. M., } \\
\text { Pompeu, A. B. G., Santos, T. L., } \\
\text { Pinto, K. H. M. S., Fonseca, E. C. } \\
\text { A., Barros, C. C. R., \& Medeiros, } \\
\text { T. S. P. }\end{array}$ \\
\hline $\begin{array}{l}\text { Physis Revista de Saúde } \\
\text { Coletiva. }\end{array}$ & $\begin{array}{l}\text { Barreiras à realização do exame } \\
\text { Papanicolau: perspectivas de usuárias e } \\
\text { profissionais da Estratégia de Saúde da } \\
\text { Família da cidade de Vitória da } \\
\text { Conquista-BA. }\end{array}$ & Aguilar, R. P., \& Soares, D.A. \\
\hline Rev Bras Ginecol Obstet. & $\begin{array}{l}\text { Por que a prevalência de resultados } \\
\text { citopatológicos do rastreamento do } \\
\text { câncer do colo do útero pode variar } \\
\text { significativamente entre duas regiões do } \\
\text { Brasil? }\end{array}$ & $\begin{array}{l}\text { Discacciati, M. G., Barboza, B. M. } \\
\text { S., \& Zeferino, L. C. }\end{array}$ \\
\hline $\begin{array}{l}\text { Revista da Escola de } \\
\text { Enfermagem da USP. }\end{array}$ & $\begin{array}{l}\text { Revisão integrativa: conceitos e } \\
\text { métodos utilizados na enfermagem. }\end{array}$ & $\begin{array}{l}\text { Soares, C. B,, Hoga, L. A. K., } \\
\text { Peduzzi, M., Sangaleti, C., } \\
\text { Yonekura, T., \& Silva, D. R. A. D. }\end{array}$ \\
\hline $\begin{array}{l}\text { Repositório Institucional } \\
\text { Tiradentes. }\end{array}$ & $\begin{array}{l}\text { Percepção das mulheres ao exame } \\
\text { citopatológico do colo uterino. }\end{array}$ & $\begin{array}{l}\text { Nascimento, A. M. B., Palmeira, M. } \\
\text { B. N. N. M. S., \& Carvalho, S. Q. } \\
\text { S. }\end{array}$ \\
\hline Ministério da Saúde. & $\begin{array}{l}\text { Portaria N 2.436, de } 21 \text { de setembro de } \\
2017 .\end{array}$ & Ministério da Saúde. \\
\hline Revista Ciência Plural. & $\begin{array}{l}\text { Exame citopatológico do colo uterino: } \\
\text { descrição dos principais indicadores em } \\
\text { um minicípio nordestino. }\end{array}$ & Rodrigues, M., \& Morais, M. \\
\hline
\end{tabular}

Fonte: Autores. 
Quadro 3. Resumo dos estudos pesquisados envolvendo cenários, metodologia e ano.

\begin{tabular}{|c|c|c|}
\hline Cenários & Metodologia & Ano \\
\hline Comunidade de dendê & Pesquisa descritiva, qualitativa & 2014 \\
\hline $\begin{array}{l}\text { USF Nossa Senhora de Fátima } \\
\text { situada no município de Senhor do } \\
\text { Bonfim-BA }\end{array}$ & Estudo quantitativo & 2014 \\
\hline $\begin{array}{l}\text { USF do município de Senhor do } \\
\text { Bomfim-BA }\end{array}$ & Estudo Qualitativo & 2015 \\
\hline Escola pública do ensino médio & Relato de experiência & 2015 \\
\hline $\begin{array}{l}\text { Mulheres que não realizaram o } \\
\text { exame citológico. }\end{array}$ & Revisão de literatura & 2014 \\
\hline $\begin{array}{l}\text { Contribuir com a organização da } \\
\text { Rede de Atenção ao Câncer do } \\
\text { Colo do Útero e da Mama no } \\
\text { Sistema Único de Saúde (SUS). }\end{array}$ & Manual do Ministério da Saúde. & 2013 \\
\hline $\begin{array}{l}\text { Mulheres quilombolas residentes } \\
\text { em Vitória da Conquista, Bahia. }\end{array}$ & $\begin{array}{l}\text { Estudo transversal de base } \\
\text { populacional, }\end{array}$ & 2014 \\
\hline $\begin{array}{l}\text { Avaliar integralidade na dimensão } \\
\text { do acesso aos serviços de } \\
\text { prevenção, diagnóstico e tratamento } \\
\text { do câncer de colo do útero. }\end{array}$ & Metodologia Quanti-qualitativa & 2014 \\
\hline $\begin{array}{l}\text { Unidade Básica de Saúde não } \\
\text { identificada. }\end{array}$ & Relato de experiência & 2020 \\
\hline $\begin{array}{l}\text { Atuação da enfermagem nas } \\
\text { Unidades Básicas de Saúde. }\end{array}$ & $\begin{array}{l}\text { Revisão da Literatura com } \\
\text { abordagem qualitativa e } \\
\text { exploratória. }\end{array}$ & 2020 \\
\hline $\begin{array}{l}\text { Unidades Básicas de Saúde da } \\
\text { cidade de Tucurui - PA. }\end{array}$ & $\begin{array}{l}\text { Abordagem por meio da teoria da } \\
\text { problematização. }\end{array}$ & 2020 \\
\hline $\begin{array}{l}\text { Cidade de Vitória da Conquista- } \\
\text { BA. }\end{array}$ & Análise categorial temática & 2015 \\
\hline Cidade do Rio de Janeiro & $\begin{array}{l}\text { Análise do Sistema de Informação } \\
\text { do Câncer do Colo de Útero. }\end{array}$ & 2014 \\
\hline $\begin{array}{l}\text { Estudos sobre teorias e } \\
\text { metodologias de RI. }\end{array}$ & $\begin{array}{l}\text { Estudo de RI desenvolvido } \\
\text { conforme a proposição de duas } \\
\text { autoras americanas. }\end{array}$ & 2014 \\
\hline $\begin{array}{l}\text { Unidade Básica de Saúde localizada } \\
\text { no município de Maceió. }\end{array}$ & $\begin{array}{l}\text { Trata-se de uma pesquisa } \\
\text { exploratória e descritiva, com } \\
\text { abordagem qualitativa. }\end{array}$ & 2020 \\
\hline $\begin{array}{l}\text { Aprova a Política Nacional de } \\
\text { Atenção Básica, estabelecendo a } \\
\text { revisão de diretrizes para a } \\
\text { organização da Atenção Básica, no } \\
\text { âmbito do Sistema Único de Saúde } \\
\text { (SUS). }\end{array}$ & Portaria do MS & 2017 \\
\hline Município de Mossoró/RN. & $\begin{array}{l}\text { Levantamento } r \text { retrospectivo } \\
\text { analítico dos }\end{array}$ & 2020 \\
\hline
\end{tabular}

Fonte: Autores. 
Quadro 4. Resumo dos estudos pesquisados envolvendo objetivos e resultados.

\begin{tabular}{|l|}
\hline Objetivos \\
\hline $\begin{array}{l}\text { Avaliar a prevalência de realização da prevenção do câncer do } \\
\text { colo uterino e fatores associados à coleta anual. }\end{array}$ \\
\hline $\begin{array}{l}\text { Descrever o conhecimento e a prática sobre papanicolau das } \\
\text { mulheres entre } 25 \text { a } 59 \text { anos atendidas pela estratégia de saúde } \\
\text { da família. }\end{array}$ \\
\hline $\begin{array}{l}\text { Avaliar a percepção de mulheres sobre o câncer do colo do } \\
\text { útero, através da prática de educação popular como instrumento } \\
\text { participativo. }\end{array}$ \\
\hline $\begin{array}{l}\text { Relatar a experiência de acadêmicas do curso de enfermagem } \\
\text { ao participarem de um projeto de extensão. }\end{array}$ \\
\hline
\end{tabular}

Identificar na bibliografia o conhecimento das mulheres sobre a importância do exame preventivo do colo do útero (Papanicolau).

Contribuir com a organização da Rede de Atenção ao Câncer do Colo do Útero e da Mama no Sistema Único de Saúde (SUS) considerando a Política Nacional de Atenção Básica, a Política Nacional de Humanização e a Política Nacional de Atenção Oncológica.

Analisar os fatores associados a não realização do exame de Papanicolau entre as mulheres quilombolas residentes em Vitória da Conquista, Bahia.

Avaliar integralidade na dimensão do acesso aos serviços de prevenção, diagnóstico e tratamento do câncer de colo do útero.

Destacar o exame citopatológico como principal estratégia para detectar lesões no colo do útero precocemente, através da técnica de coleta adequada.

Destacar a importância da atuação do enfermeiro em ações de orientação e informação das usuárias das Unidades Básicas de Saúde, sobre a representatividade da prevenção do câncer do colo de útero para a qualidade de vida e saúde das mesmas, acrescendo a necessidade de retornarem para a retirada do resultado do exame citopatológico.

Conhecer as barreiras que levam mulheres em idade fértil da cidade de Vitória da Conquista-BA a não realizarem o exame Papanicolau, na perspectiva das próprias mulheres e dos profissionais de saúde.

Fornecer bases científicas para os enfermeiros da atenção básica da cidade de Tucurui-PA, através de treinamentos em parceria com a secretaria de saúde, no intuito de esclarecer quanto as responsabilidades gerais desses profissionais, tendo em vista a complexidade do exame.

Analisar a prevalência dos resultados citopatológicos empregados para o rastreamento do câncer do colo do útero em relação à faixa etária da mulher e ao tempo de realização do último exame, no Rio de Janeiro e Maceió, pelo Sistema Único de Saúde.

Identificar e analisar conceitos e métodos indicados para desenvolver uma RI na enfermagem.

\section{Resultados}

A proporção de mulheres que relatou coleta anual foi de 59,3\%.

Demostram que ainda existem mulheres que não realizam o papanicolaou regularmente e, principalmente, desconhecem a finalidade do procedimento.

Foi possível perceber que as participantes reconhecem a importância, foi mencionado sentimento de insegurança e vergonha na realização do exame.

A experiência vivida resultou na constatação de que são necessárias ações educativas contínuas para incentivar as mulheres à realização do exame.

O câncer de colo uterino é uma das raras moléstias malignas curáveis em $100 \%$ dos casos, quando diagnosticada precocemente através do exame de Papanicolau.

Orientar a atenção às mulheres subsidiando tecnicamente os profissionais da Atenção Básica em saúde, disponibilizando conhecimentos atualizados de maneira acessível.

De 348 mulheres incluídas na análise, 27,3\% afirmaram nunca ter realizado o Papanicolau.

Houve baixa cobertura do exame de Papanicolau, possivelmente devido à busca ativa insuficiente e à dificuldade de agendamento das consultas na atenção básica.

A coleta para o exame do Papanicolau foi realizada, onde no colo do útero era possível observar uma lesão infiltrativa de coloração vermelha com áreas negras aparentando necrose que sangrava espontaneamente.

É imprescindível a atuação da enfermagem nas Unidades Básicas de Saúde, realizando ações preventivas do câncer do colo de útero, com a conscientização da população feminina, sobre a importância do exame Papanicolau ou citopatológico e a retirada do resultado.

Foi aplicada análise categorial temática, que revelou as seguintes barreiras para não realização do exame: conhecimento insuficiente, sentimentos negativos, falta de atitude, aspectos vinculados aos serviços de saúde e inserção da mulher no mercado de trabalho. A congruência e incongruência de significados emergidos possibilitarão um caminho que transforme a prática do profissional de saúde e atendimento das necessidades dessas mulheres.

A realização do treinamento seguiu como planejado, sendo possível visualizar durante os debates a complexidade do problema. Isso permitiu concluir que o trabalho contínuo com a equipe de enfermagem e o próprio interesse dos profissionais em buscar conhecer profundamente o conteúdo do câncer de colo uterino e o exame preventivo ainda são fundamentais para o empoderamento profissional de forma segura e eficaz.

No Rio de Janeiro, predominaram exames citopatológicos realizados com intervalo de um e dois anos e, em Maceió, controles com intervalo de um e três anos. As mulheres atendidas no Maceió eram mais velhas do que aquelas do Rio de Janeiro. A prevalência do resultado citopatológico carcinoma escamoso invasor foi semelhante entre os municípios, porém todos os demais resultados apresentaram prevalência superior no Rio de Janeiro: atipias de significado indeterminado em células escamosas.

Os resultados indicam que os estudos foram desenvolvidos majoritariamente nos EUA; é possível ter várias questões de pesquisa ou hipóteses e incluir investigações desenvolvidas através de diferentes referenciais teóricos e metodológicos. 


\begin{tabular}{|c|c|}
\hline $\begin{array}{l}\text { A pesquisa teve como objetivo identificar a percepção das } \\
\text { mulheres sobre a realização de exame citopatológico do colo } \\
\text { uterino. }\end{array}$ & $\begin{array}{l}\text { Diante das falas das } 10 \text { mulheres que participaram deste estudo, } \\
\text { foi possível identificar } 3 \text { categorias relacionadas ao exame } \\
\text { citopatológico: prevenção, desconforto associado a vergonha e o } \\
\text { esclarecimento de dúvidas. Durante as entrevistas, elas } \\
\text { demonstraram insegurança em responder com exatidão os } \\
\text { motivos que a levam a realizar o exame. }\end{array}$ \\
\hline $\begin{array}{l}\text { Aprova a Política Nacional de Atenção Básica, estabelecendo a } \\
\text { revisão de diretrizes para a organização da Atenção Básica, no } \\
\text { âmbito do Sistema Único de Saúde (SUS). }\end{array}$ & Cumprimento da portaria. \\
\hline $\begin{array}{l}\text { O objetivo desse estudo foi descrever os resultados dos exames } \\
\text { citopatológicos em mulheres de } 25 \text { a } 64 \text { anos no Município de } \\
\text { Mossoró/RN. }\end{array}$ & $\begin{array}{l}\text { Um total de } 46606 \text { casos foram incluídos e analisados. Houve } \\
\text { uma tendência de aumento na realização dos testes ao longo dos } \\
\text { anos. } 83,8 \% \text { dos casos representam células escamosas atípicas de } \\
\text { significado indeterminado (ASC-US). } 70 \% \text { das lesões } \\
\text { intraepiteliais escamosas (LIS) foram diagnosticadas como sendo } \\
\text { de baixo grau (LISL). }\end{array}$ \\
\hline
\end{tabular}

Fonte: Autores.

A Atenção Básica é a porta de entrada do usuário aos serviços oferecidos pelo Sistema Único de Saúde, sendo descentralizada, próxima as famílias e suas condições de vida. São diversas as ações da Atenção Básica para fortalecimento da saúde da comunidade, entre elas estão à prevenção do câncer de colo de útero e de mama por serem respectivamente os cânceres que mais atingem as mulheres no Brasil e até no mundo. O câncer de colo uterino é a segunda maior causa de morte, perdendo apenas para o câncer de mama (Brasil, 2013; Salvi et al., 2020).

O câncer de colo de útero é diagnosticado através do exame citopatológico, o qual é um exame extremamente confiável em que pode reduzir o número de casos desse câncer em mulheres que o realizam regularmente. Os autores destacam a importância da realização desse exame para detecção precoce do CCU, de maneira que a qualidade de vida da mulher seja preservada através dessa ação de prevenção e promoção da saúde, além disso, os autores também concordam no fato de que este exame deve ser de fácil acesso na UBS, não tendo nenhum tipo de dificuldade para sua realização, por isso o enfermeiro deve estar sempre se atualizando e renovando as estratégias para melhora na aceitação do Papanicolau pela comunidade, levando a consideração a realidade social de cada grupo (Oliveira et al., 2014; Brasil, 2013; Souza et al., 2014).

Uma pesquisa realizada com mulheres que estudam numa escola pública revelou que ainda existem muitas dúvidas sobre o câncer de colo de útero, assim como sobre o exame citológico e outras diversas questões sobre sexualidade, principalmente relacionadas ao próprio corpo feminino. Assim, identificou-se a importância de atividades educativas sobre esses temas que são essenciais, pois estão dentro do cotidiano de todas as mulheres (Valente et al., 2015).

Já a pesquisa realizada através de entrevistas domiciliares com mulheres de idade fértil em Fortaleza CE, identificou que as mulheres que apresentam mais chance de realizar o exame citopatológico regularmente são aquelas que tem trabalho fixo com boa remuneração e aquelas que já tiveram pelo menos o seu primeiro filho (Falcão et al., 2014; Andrade et al., 2014).

Para reafirmar esses dados, Nascimento e colaboradores (2020) identificaram através de sua pesquisa que mesmo com tanta informação disseminada nas Unidades de Saúde, ainda existem diversas mulheres que não realizam o exame citológico regularmente e ainda desconhecem o que é o procedimento e sua importância. Por isso, vale enfatizar que as atividades educativas nas Unidades Básicas de Saúde são essenciais para informar e sensibilizar as usuárias sobre o assunto (Nascimento et al., 2020).

Andrade et al (2014) enfatizam que a maior parte das mulheres que não aderiram a realização do exame citológico são aquelas que nunca frequentaram a escola, que possui quatro ou mais filhos, não utilizam nenhum método anticoncepcional e tem um conhecimento ineficiente sobre a execução do exame.

Existem vários fatores que dificultam a realização do exame citológico para mulheres que necessitam ser atendidas na Atenção Básica, entre eles foi identificado a busca ativa ineficiente dessas mulheres na comunidade e a dificuldade para marcar o referido exame nas unidades. A cobertura do exame foi maior em mulheres mais jovens, sendo considerado difícil o acesso 
das mulheres com mais idade. Também foi possível identificar que todas as citologias alteradas foram encaminhadas para a biópsia, a qual também teve resultado alterado, e os diagnósticos mais graves encontrados nas citologias e nas biópsias foram de mulheres mais velhas (Silva et al., 2014; Lopes; Lopes, 2020).

A partir desses achados, observa-se que há uma dificuldade para as mulheres com mais idade realizarem o exame citológico regularmente e das que fizeram o exame, em grande parte detectou-se resultados alterados. Muitas vezes, mulheres mais velhas acham que não precisam mais realizar o exame por diversos motivos que podem ser o fato de não terem mais relação sexual ou estarem na menopausa, porém mesmo com essas situações, todas as mulheres devem manter o exame atualizado anualmente ou conforme necessidade.

O acolhimento à mulher na Atenção Básica é indispensável para a boa aceitação do exame Papanicolau, humanizando o processo e facilitando a participação coletiva nas práticas de saúde. Sendo assim, os profissionais têm o dever de estimular a adesão das mulheres ao exame citopatológico de forma a incentivar ao cuidado com sua própria saúde (Brasil, 2013; Silva et al., 2020).

Uma pesquisa realizada em Vitória da Conquista - Bahia, analisou 348 mulheres, dentre essas 27,3\% afirmaram que nunca realizaram o exame citológico devido a alguns fatores que envolvem falta de instrução, não ter um companheiro, procurar atendimento em unidades que não correspondem à área de sua residência e foram identificados que as idades que envolvem fatores de negação são entre 18-29 anos e 50-59 anos (Oliveira et al., 2014).

As baixas condições socioeconômicas das mulheres contribuem para a falta de conhecimento sobre o exame ou até mesmo contribuem para a negação de execução do mesmo. A ausência de sinais e sintomas sugestivos de inflamação que incomodem a mulher também é um fator que pode leva-la a achar que não é necessário realizar o exame pois aparentemente está saudável, adiando cada vez mais o método mais fácil para diagnosticar precocemente o câncer de colo de útero (Santos et al., 2014).

Destaca-se que a Atenção Básica ainda possui muitas fragilidades no que tange a busca ativa de mulheres que iniciaram a vida sexual para a realização do exame de prevenção do câncer de colo do útero, contribuindo assim para o aumento no número de casos desse câncer em mulheres de qualquer idade (Oliveira et al., 2014; Silva et al., 2020).

Para melhorar e aumentar o número de mulheres a procurar o exame regularmente é necessário aumentar a oferta de vagas do mesmo para facilitar a marcação, assim como realizar atividades educativas é de extrema importância principalmente quando envolve todos os profissionais de saúde do setor, assim como a comunidade específica (Santos et al., 2014).

A interação entre profissionais de saúde dentro das Unidades Básicas de Saúde é imprescindível para o sucesso nas palestras educativas, é necessário enfatizar o problema de maneira didática, que não inflija nas crenças do outro, mas que sensibilize a população feminina da importância do exame citopatológico para sua saúde, isso irá contribuir para a redução do número de morbimortalidade materna por esse motivo.

As atividades educativas precisam acontecer de forma que chamem a atenção das mulheres com exemplos práticos do cotidiano delas para que seja mais eficaz o entendimento. Sendo assim, o enfermeiro como líder na Equipe de Saúde da Família deve estimular os outros profissionais para o acolhimento da mulher na unidade de forma que facilite o próximo contato para que haja a sensibilização acerca do exame Papanicolau (Silva et al., 2020; Santos et al., 2014).

\section{Conclusão}

Observa-se que mesmo diante de tanta informação que todos os indivíduos têm acesso nos dias atuais, existem inúmeras mulheres que não realizam o exame citológico regularmente por medo ou falta de informação. Foram destacados nesse estudo através da pesquisa bibliográfica que a maior parte da população feminina que mantém em dia o exame são mais jovens, mas aquelas com mais idade que o realizaram foi detectado, em sua maioria, alterações importantes. 
Dessa forma, compreende-se que existem diversos motivos pelos quais dificultam a adesão das mulheres ao exame citopatológico, porém cabe a equipe de saúde sensibilizar a mulher quanto a sua importância, enfatizando os benefícios de detectar precocemente um câncer de colo de útero para um prognóstico favorável e redução na morbimortalidade.

Por fim, a presente pesquisa poderá abrir espaço para trabalhos futuros alinhando as dificuldades que as mulheres enfrentam para realizar o exame citológico nas Unidades Básicas de Saúde em todo o Brasil ou em algum lugar específico, bem como a importância desse exame para a prevenção do colo de útero e outras patologias.

\section{Referências}

Aguilar, R. P., \& Soares, D. A. (2015). Barreiras à realização do exame Papanicolau: perspectivas de usuárias e profissionais da Estratégia de Saúde da Família da cidade de Vitória da Conquista-BA. [Manuscrito publicado, Physis Revista de Saúde Coletiva]. https://.org/10.1590/S0103-73312015000200003 .

Andrade, M. G., Almeida, M. M. G., Araújo, T. M., \& Santos, K. O. B. (2014). Fatores associados a não adesão ao Papanicolau entre mulheres atendidas pela Estratégia Saúde da Família em Feira de Santana, Bahia. [Manuscrito publicado, Epidemiologia Serv. Saúde]. https://.org/10.5123/S167949742014000100011

Brasil. (2013). Controle dos cânceres de colo de útero e de mama. Departamento de Atenção Básica. Ministério da Saúde.

Brasil. (2017). Portaria N 2.436, de 21 de setembro de 2017. Aprova a Política Nacional de Atenção Básica, estabelecendo a revisão de diretrizes para a organização da Atenção Básica, no âmbito do Sistema Único de Saúde (SUS). Ministério da Saúde.

Discacciati, M. G., Barboza, B. M. S., \& Zeferino, L. C. (2014). Por que a prevalência de resultados citopatológicos do rastreamento do câncer do colo do útero pode variar significativamente entre duas regiões do Brasil? [Manuscrito publicado, Revista Brasileira de Ginecologia e Obstetrícia]. https://.org/10.1590/S0100-7203201400050002.

Falcão, G. B., Ibiapina, F. L. P., Feitosa, H. N., Feitosa, T. S., Lacerda, P. D., Braga, J. U., \& Carvalho, F. H. C. (2014). Fatores associados à realização de citologia para prevenção de câncer do colo uterino em uma comunidade urbana de baixa renda. [Manuscrito publicado Cadernos de Saúde Coletiva]. https://.org/10.1590/1414-462X201400020009.

Lopes, P., \& Lopes, A. (2020). A importância do exame citopatológico nas Unidades Básicas de Saúde: A atuação do enfermeiro. [Manuscrito publicado Revista Intersaúde].

Nascimento, A. M. B., Palmeira, M. B. N. N. M. S., \& Carvalho, S. Q. S. (2020). Percepção das mulheres ao exame citopatológico do colo uterino. [Manuscrito publicado, Repositório Institucional Tiradentes].

Oliveira, M. V., Guimarães, M. D. C., \& França, E. B. (2014). Fatores associados a não realização de Papanicolau em mulheres quilombolas. [Manuscrito publicado, Ciência \& Saúde Coletiva]. https://.org/10.1590/1413-812320141911.15642013.

Rodrigues, M., \& Morais, M. (2020). Exame citopatológico do colo uterino: descrição dos principais indicadores em um município nordestino. [Manuscrito publicado, Revista Ciência Plural]. https://.org/10.21680/2446-7286.2020v6n3ID20698.

Soares, C. B., Hoga, L. A. K., Peduzzi, M., Sangaleti, C., Yonekura, T., \& Silva, D. R. A. D. (2014). Revisão integrativa: conceitos e métodos utilizados na enfermagem. [Manuscrito publicado, Revista da Escola de Enfermagem da USP]. https://.org/10.1590/S0080-6234201400002000020.

Salvi, E. S. F., Pompermaier, C., Floriani, F., Barrionuevo, V., \& Block, J. C. (2020). Exame Citopatológico. Anuário pesquisa e extensão Unoesc Xanxerê.

Souza, K. R., Paixão, G. P. N., Almeida, E. S., Sousa, A. R., Lirio, J. G. S., \& Campos, L. M. (2014). Educação popular como instrumento participativo para a prevenção do câncer ginecológico: percepção de mulheres. [Manuscrito publicado, Revista Cuidarte]. https://.org/10.15649/cuidarte.v6i1.129.

Silva, K. B., Bezerra, A. F. B., Chaves, L. D. P., \& TanakA, O. Y. (2014). Integralidade no cuidado ao câncer do colo do útero: avaliação do acesso. [Manuscrito publicado, Revista de Saúde Pública]. https://.org/10.1590/S0034-8910.2014048004852.

Silva, N. F. C. P., Silva, C. M., Pompeu, A. B. G., Santos, T. L., Pinto, K. H. M. S., Fonseca, E. C. A., Barros, C. C. R., \& Medeiros, T. S. P. (2020). Training of primary care nurses regarding cervical cytopathological examination. 10.34119/bjhrv3n5-303.

Santos, M. A., Audickas, R. C., Coutinho, S. C., Silva, J., \& Souza, L. N. (2014). A importância da prevenção do câncer do colo uterino: em pauta o exame de Papanicolau. https://.org/10.24276/rrecien2358-3088.2014.4.12.15-20.

Valente, C. A., Andrade, V., Soares, M. B. O., \& Silva, S. R. (2015). Atividades educativas no controle do câncer de colo do útero: relato de experiência. [Manuscrito publicado, R. Enferm. Cent. O. Min]. https://.org/10.19175/recom.v5i3.576. 